\title{
Models of Virulent Phage Growth with application to Phage Therapy
}

\author{
Hal L. Smith*
}

February 7, 2008

\begin{abstract}
We modify existing models of bacteriophage growth on an exponentially growing bacterial population by including (1) density dependent phage attack rates and (2) loss to phage due to adsorption to both infected and uninfected bacteria. The effects of these modifications on key pharmacokinetic parameters associated with phage therapy are examined. More general phage growth models are explored which account for infection-age of bacteria, bacteria-phage complex formation, and decoupling phage progeny release from host cell lysis.
\end{abstract}

Keywords: phage therapy, infection-age structure, multiple adsorptions, bacteriaphage complex, passive therapy, active therapy, proliferation threshold.

\section{Introduction}

As pathogenic bacteria have increasingly become resistant to our arsenal of antibiotics, there has been renewed interest in the use of bacteriophages to control bacterial infections $[13,11,12,14,15,23]$. Bacteriophages, phages for short, are viruses which prey on bacteria. Almost as soon as they were discovered there was interest in using them to control infections and bacterial contamination. The history of early attempts to use them for such purposes during the last century is fascinating $[13,12,6]$. It is not hard to see the

\footnotetext{
${ }^{*}$ Supported by NSF Grant DMS 0414270, Department of Mathematics, Arizona State University, Tempe, AZ
} 
potential in phage therapy for unlike chemotherapy, which simply results in the death of a susceptible bacteria, phage therapy results in the death of the host cell and the release of hundreds more lethal phage. The author found the review articles $[13,12]$ on phage therapy useful.

Mathematical modeling has long played a significant role in the study of phage-bacterial interactions for ecological reasons $[2,22,17,9]$ as well as for medical ones $[3,11,12,10,14,15,23]$. See also the additional references in these papers. The reasons for this are obvious-among them being the difficulty of carrying out controlled experiments in vivo and the novelty of a self-replicating therapeutic agent.

It will be useful to briefly review the life cycle of a virulent phage and some of the associated terminology for later use. Typically, phage specialize to attack only one or a few strains of bacterial host whose cell surface contains an appropriate binding site. Phage attach to a preferred binding site, then inject their genetic material, DNA or RNA depending on the phage, and perhaps some enzymes into the cell which thereafter is called an infected cell. In the case of virulent (also called lytic) phage, the infected host cell machinery is then immediately co-opted to make new phage particles which are subsequently released in a burst when phage enzymes cause the host cell to lyse and the cycle repeats. The latent period is the time between phagehost binding and subsequent release of phage at cell lysis, usually on the order of 20 minutes to an hour depending on the host-phage system. The burst size, ranging between several to thousands, is a measure of the average number of phage progeny resulting from a single infected host cell and also depends on the host-phage system.

This paper addresses some issues that seem not to have been explored in the mathematical modeling of bacteriophage growth that may be important in phage therapy. First, one finds that mass action kinetics, e.g. $b x v$, where $x$ denotes concentration of uninfected bacteria and $v$ is concentration of phage, is invariably used to model both the phage attack rate on uninfected bacteria and the rate of loss of free phage due to attachment $[9,10,14,15,23]$. Heinemann et al. [23] experimentally measured the "adsorption rate" $b$ in the context of the rate of loss of phage (number of adsorbed phage per freephage per bacterium per minute) and found a wide variation of values and noted that it decreased as the sum of phage and bacterial densities $(x+y+v)$ increased, where $y$ is the density of infected bacteria.

In this paper, we will argue that the phage attack rate and the rate of phage loss due to attachment are distinct. As the former involves attachment 
and injection of the one primary (first to inject) phage while the latter takes account of all secondary phage that attach to a cell, this should not be unexpected. We propose that the phage attack rate deviates from $b x v$ when phage densities are large due to higher likelihood of multiple phage binding to a cell between the time of initial binding and lysing and therefore to a lower impact per phage particle. Mathematically, this will be achieved here not by making $b$ dependent on the densities but by adding an extra multiplicative term to the phage attack rate which depends on the phage density. Specifically, our analysis leads to the reduced phage attack rate

$$
\frac{b x v}{F_{N}(c v)}, \quad c=b / \rho
$$

where $1 / \rho$ denotes the injection time, the time between binding of a phage to a host bacteria and subsequent injection of genetic material into the host, $N$ denotes the number of binding sites for phage per host, and

$F_{N}(u)=1+\frac{u}{1+u}+\frac{u^{2}}{(1+u)(2+u)}+\cdots+\frac{u^{N}}{(1+u)(2+u) \cdots(N-1+u) N}$

Observe that $F_{N}(u)>1$ so the attack rate is strictly less than bxv. Despite appearances, $F_{N}(u)$ depends rather weakly on $N ; F_{3}(u)$ is a good approximation of $F_{100}(u)$ on $0<u<5$. To lowest order, $F_{N}(u) \approx 1+u$ so the effect of the term $c v$ in (1) is non-negligible precisely when $b v \times \frac{1}{\rho}$ is nonnegligible compared to one. $b v / \rho$ gives the number of potential irreversible phage attachments that could be formed with a typical host cell during the injection time. A rough estimate of $c=O\left(10^{-8}\right)$ for a strain of E. coli and phage implies that the term $c v$ in (1) is significant when $v \geq O\left(10^{7}\right)$, well within the range used in experimental and theoretical studies.

Secondly, it seems to us that the rate of loss of phage is underestimated in existing models and moreover that the effect of this underestimation may be significant for bacteriophage therapy. For example, if we assume that phage cannot detect the state (uninfected or infected) of the host cell to which it binds then one should not ignore the loss of phage due to "wasted attacks" on already infected hosts. We take into account that a host cell has a multiplicity of potential phage binding sites on its surface, more than one of which may be simultaneously bound by phage. This leads, with good approximation, to the expression

$$
-b v(x+y)
$$


for the rate of phage loss due to attachment. As a result our model differs from others in the literature in that the phage loss rate due to attachment differs from the phage attack rate on host.

Finally, most existing models assume either an exponentially distributed latent period [14, 15], leading to ordinary differential equations, or assume a fixed-length latent period which results in delay differential equations $[9,10$, 23]. Here, in an appendix, we explore a more general model where infected cells are structured by age-since-injection and where the release rate of phage progeny may be either a continuous "budding off" or an abrupt burst at host lysis typical of virulent phage. We also allow for variable phage progeny size by decoupling cell death from the release of progeny. This structured model may lead to ordinary differential equations, to delay equations, or to more general integro-differential equations depending on whether the infected cell mortality rate is independent of age-since-injection, sharply dependent on it, or a more smooth nonconstant function of it. However, much of our effort is devoted to a delay differential equation model resulting from the assumption of a fixed-length latent period followed by a discrete burst of phage. This model is similar to ones considered by Lenski and Levin [10] and by Beretta and Kuang [2] except for the modifications already noted. Our treatment of the initial conditions and their effect during the initial latent period is more natural than in $[10,2]$ and it facilitates the consideration of a proliferation threshold for phage therapy.

We show that the density dependent attack rate (1) and the modified rate of phage loss due to attachment (2) result in a potentially significant modifications in key pharmacokinetic quantities associated with active phage therapy first identified by Payne and Jansen $[14,15]$. Unlike passive therapy which relies on a massive dose of phage to kill bacteria in only one phage generation, active therapy does not need such large dose since it relies on second and third generation phage for its success. Payne and Jansen argued for the existence of a threshold number of uninfected host cells required to support the amplification in phage numbers from one phage generation to the next (phage reproductive number exceeds one). This idea has generated some controversy in the field $[7,8,13,16]$ due to a misunderstanding of its meaning. However, it is certainly valid for the mathematical models of phage growth treated in $[14,23]$. We derive a threshold condition for phage proliferation which reduces to one comparable to Payne and Jansen's when total bacterial density is not too large but changes character when densities become large. 


\section{Phage Growth on an Exponentially Grow- ing Host Population: Fixed-Length Latent Period}

In the appendix we derive a general model of phage growth which includes the one described below as a special case. Here, we make the following assumptions:

(a) bacteria first injected by phage at time $t-\tau$ lyse (die) at time $t$.

(b) uninfected host cells grow at rate $a$; infected cells do not grow.

(c) free phage, those unattached to host cells, decay or washout at rate $m$.

(d) bacteria are removed by washout or death unrelated to phage at rate $p$.

(e) phage do not distinguish between infected and uninfected cells in regard to attachment and injection.

(f) the rate of release of phage progeny from an infected host of infectionage (time since injection) $s \in[0, \tau]$ is $\eta(s)$. In particular, it is independent of the number of phage injections.

In other words, we assume the latent period has duration precisely $\tau$ units of time as in $[2,23,10]$; this is relaxed in the more general model in the appendix. As we are primarily motivated by applications to phage therapy where one is interested in treating the initial phase of a bacterial infection which, in the worst case, is characterized by an exponentially growing pathogen, the model equations do not include density effects on host growth such as in $[10,2]$. Payne and Jansen $[14,15]$ assume that infected host grow at the same rate as uninfected ones but we follow Heinemann et al. [23] and Abedon et al. [1] who suggest that infected host cells do not grow. According to (f), the integral

$$
L=\int_{0}^{\tau} \eta(s) d s
$$

gives the phage progeny from an infected host assuming that it survives the latent period. The general release rate $\eta(s)$ easily accommodates both what is sometimes called "budding" of phage progeny from a living host cell as well 
as a "burst" of phage progeny released at cell lysis. Assumption (e) is used by Schrag and Mittler in [18] in an ecological setting. We are unaware of any evidence supporting or refuting it. Our assumption in (f) that the number of progeny is independent of the number of phage injections is consistent with observations of Stent [20], pg 74, who remarks that "latent period and burst size do not, however, depend in any very striking way on the number of phage particles with which each bacterial cell has been infected". However, he later mentions lysis inhibition that occurs in the infected host of certain T-even phage where superinfection late in the latent stage may substantially prolong the latent stage and enhance the burst size. See also [1]. Hypothesis (d) is rather standard $[14,23]$.

Let $x$ denote the density of uninfected host bacteria, the density of phageinfected bacteria by $y$, and phage density by $v$. The expression (1) is used for phage attack rate rather than the usual mass action rate $b x v$ for the reasons described in the introduction. The corresponding loss rate for phage due to attachment to host cells is modeled by (2) to account for wasted attachments following (e) above. We stress that both these rates are derived in the appendix.

Initial conditions at time $t=0$ must take account of the infection-age of the infected cells since these cells may have been infected at different times in the past. Moreover, it is reasonable to assume that the initial set of infected cells were obtained in a manner independent of the initial set of uninfected cells and phage. Therefore, we prescribe the initial uninfected host density $x(0)$, the initial phage density $v(0)$, and the initial distribution of infected cells:

$$
U_{0}(s), 0 \leq s \leq \tau
$$

where $s$ denotes the age since infection (injection). Thus, for $0<c<d<\tau$, $\int_{c}^{d} U_{0}(s) d s$ denotes the number of infected cells with infection-age between $c$ and $d$. These cells, infected at times between $t=-d$ and $t=-c$ will, if not washed out, lyse at times between $\tau-d$ and $\tau-c$.

In our view, the manner in which initial data are formulated here is more natural than that in [2] where the past history of phage and uninfected host cells are prescribed over a latent period, assuming that the initial population of infected cells at $t=0$ arise through infection of the initial host population by the initial phage. 
For the initial latent period, the equations of the model are given by:

$$
\begin{aligned}
\frac{d x}{d t}= & a x-\frac{b x v}{F_{N}(c v)}-p x \\
y(t)= & \int_{0}^{t} \frac{b x(t-s) v(t-s)}{F_{N}(c v(t-s))} e^{-p s} d s+e^{-p t} \int_{0}^{\tau-t} U_{0}(s) d s, \quad 0 \leq t \leq \tau \\
\frac{d v}{d t}= & -b(x+y) v-m v+\int_{0}^{t} \frac{b x(t-s) v(t-s)}{F_{N}(c v(t-s))} e^{-p s} \eta(s) d s \\
& +e^{-p t} \int_{0}^{\tau-t} U_{0}(s) \eta(s+t) d s
\end{aligned}
$$

Observe that infected cells at time $t<\tau$ arise either from cells infected after $t=0$ or from surviving cells from the founding population $U_{0}$. The first integral in the equation for $y$ gives the number of cells infected after $t=0$ which survive washout to be alive at time $t$. The second gives the survivors from the founding population still alive at time $t$; clearly these must have had age less than $\tau-t$ in order to be alive at time $t$. A similar analysis explains the two integrals in the equation for phage: the first integral gives phage progeny issuing from cells infected after $t=0$, the second integral giving phage progeny issuing from survivors from the founding population of infected cells.

In all our simulations and in most experimental setups, the founding population of infected cells is taken to vanish, $U_{0}=0$, simplifying the equations during the initial latent period. Indeed, the natural initial conditions for phage therapy correspond to administering a dose of phage to an exponentially growing bacterial population that has not been exposed to phage and therefore there are initially no infected host of any age of infection. This does not mean that our care in formulating the initial conditions is wasted since we will have reason to consider nonzero $U_{0}$ in our later discussion related to phage therapy.

After the latent period, the founding population of infected cells have all lysed and the equations are simpler:

$$
\begin{aligned}
\frac{d x}{d t} & =a x-\frac{b x v}{F_{N}(c v)}-p x \\
y(t) & =\int_{t-\tau}^{t} \frac{b x(s) v(s)}{F_{N}(c v(s))} e^{-p(t-s)} d s, \quad t>\tau \\
\frac{d v}{d t} & =\int_{0}^{\tau} e^{-p s} \frac{b x(t-s) v(t-s)}{F_{N}(c v(t-s))} \eta(s) d s-b(x+y) v-m v
\end{aligned}
$$




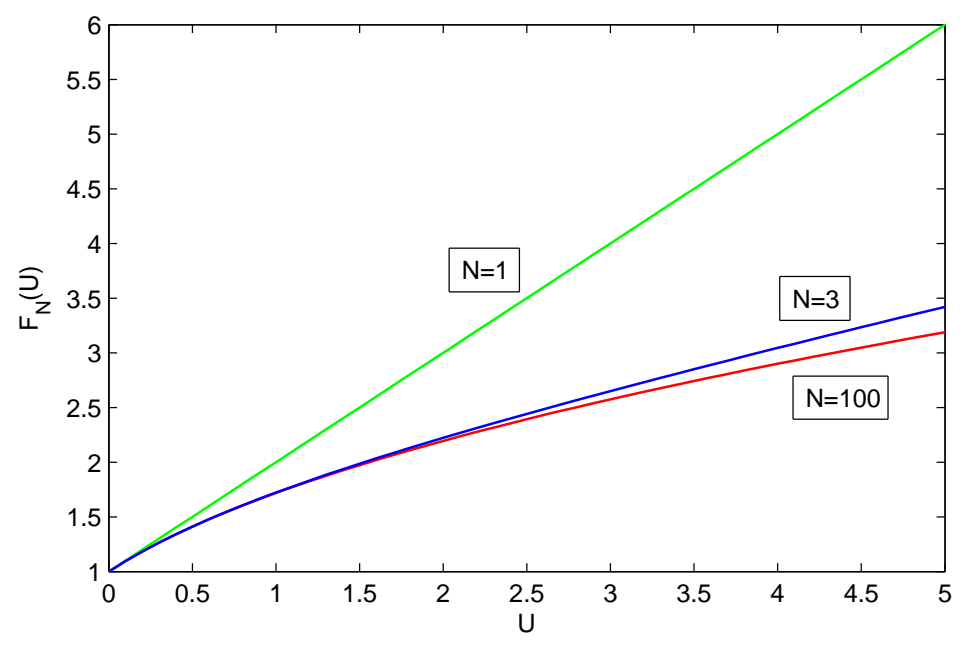

Figure 1: Plot of $F_{N}(u)$ versus $u$ for $N=1,3,100$.

Key properties of the function $F_{N}(u)$ are given in the lemma below, proved in the appendix. As Figure 1 shows, these functions depend rather weakly on $N$ for small $u$.

Lemma 1. The functions $F_{N}:[0, \infty) \rightarrow[1, \infty)$ satisfy the following:

1. $\frac{d}{d u} \frac{u}{F_{N}(u)}>0$ and $\frac{d}{d u} F_{N}(u)>0$.

2. $\frac{u}{F_{N}(u)} \rightarrow N$ as $u \rightarrow \infty$

3. $F_{\infty}(u) \leq F_{N+1} \leq F_{N}(u) \leq F_{1}(u)=1+u$

where

$$
F_{\infty}(u)=1+\sum_{n=1}^{\infty} \prod_{i=1}^{n} \frac{u}{i+u}
$$

The biological implications of these assertions are not surprising. The fact that $F_{N}(u)>1$ for $u>0$ means that the attack rate (1) is smaller than the corresponding mass action rate bxv. According to assertion 3., it increases with $N$, the number of host binding sites; more sites means the potential for more attached phage which decreases the time to injection. The first assertions ensure that the phage attack rate increases with increasing phage density $v$. The second indicates that the maximum effect of phage on the specific growth rate of bacteria $\left(x^{\prime} / x\right)$ is $b N / c$. Observe that $F_{3}(2) \approx$ 
$F_{100}(2)>2$ so the attack rate is less than half the corresponding mass action rate $b x v$ when $c v>2$.

For $t>\tau$, the $y$ equation can be differentiated to yield

$$
y^{\prime}=\frac{b x v}{F_{N}(c v)}-e^{-p \tau} \frac{b x_{\tau} v_{\tau}}{F_{N}\left(c v_{\tau}\right)}-p y
$$

where $x_{\tau}=x(t-\tau), v_{\tau}=v(t-\tau)$. As noted above, the natural initial conditions for phage therapy are to administer a dose of phage to an exponentially growing bacterial population which has not previously been exposed to phage. Thus, $U_{0} \equiv 0$ and the differentiated form of the $y$ equation during the initial latent period differs from (5) in that the second term is removed.

Well-posedness issues related to our system (3)-(4) can be treated using results in [5]. Corollary 2.2 of Chapt. 12 can be used to prove the existence and uniqueness of a maximally defined continuous solution corresponding to nonnegative initial data if, for example, $U_{0}$ is integrable and $\eta$ is essentially bounded. Easy arguments give that the solution is nonnegative and a prior bounds show that it is globally defined.

If the rate of phage progeny release is highly peaked about the age at lysis $\tau$, it is reasonable to assume that cells produce $L$-phage exactly on reaching age $\tau$ :

$$
\eta(s)=L \delta(s-\tau)
$$

where $\delta(r)$ is the Dirac impulse function with unit mass concentrated at $r=0$. In that case, the equation for phage simplifies to the following, for the latent period,

$$
\frac{d v}{d t}=-b(x+y) v-m v+L e^{-p t} U_{0}(\tau-t), \quad 0<t \leq \tau
$$

and thereafter

$$
\frac{d v}{d t}=L e^{-p \tau} \frac{b x_{\tau} v_{\tau}}{F_{N}\left(c v_{\tau}\right)}-b(x+y) v-m v, \quad t>\tau
$$

\subsection{Remarks on Long Term Dynamics}

In contrast to ecologically motivated theoretical studies of phage growth where attention has been paid to long term dynamics $[2,17]$, there has been very little consideration of long term dynamics of phage growth models aimed 
at understanding phage therapy $[14,23]$. This is probably due to a tacit assumption that the equations are only valid until such time as an immune response is mounted. Indeed, the hypothesis of exponential bacterial growth captures this focus on the short term dynamics.

Returning to the general system (3)-(4) with budding rate $\eta$, if $a>p$, which we assume hereafter, then the trivial equilibrium point $(x, y, v)=$ $(0,0,0)$ is an unstable saddle point. For if there are no virus or infected cells, then the uninfected cells grow at the exponential rate $a-p>0$. If, on the other hand, there are no uninfected cells, then any free virus and infected cells are removed at an exponential rate. Therefore, it is plausible that no solution starting with $x(0)>0$ can satisfy $x(t) \rightarrow 0, t \rightarrow \infty$. We wish to stress that this fact is common to all models of virulent phage growth in the literature not just the one treated here. It is possible to overlook this observation on viewing the simulations reported here and in $[14,23]$. Below we give a proof which carries over to these other models with only minor changes.

Proposition 1. If $a>p$, then no solution of (4) with $x(0)>0$ can satisfy $x(t) \rightarrow 0, t \rightarrow \infty$.

Proof. The assertion is obvious if $b N / c \leq a-p$ since then $x^{\prime} / x \geq 0$. Hereafter, assume that $b N / c>a-p$. If $x(t) \rightarrow 0$ as $t \rightarrow \infty$ for some non-zero solution of (4) then necessarily $y(t) \rightarrow 0$ as well. It can then be seen that $v$ must remain bounded. By standard arguments, this and the convergence of $x(t)$ implies that $x^{\prime}(t) \rightarrow 0$ and therefore, since $x(t) \neq 0, v(t) \rightarrow V_{I}>0$ where $V_{I}$ is the unique positive root of $\frac{b v}{F_{N}(c v)}=a-p$ guaranteed by Lemma 1 . The convergence of $v(t)$ implies $v^{\prime}(t) \rightarrow 0$ but this immediately leads to the contradiction that $v \rightarrow 0$.

Proposition 1 should not be construed as implying that phage therapy is doomed to failure. Obviously, our deterministic model breaks down at low densities of bacteria but aside from this, therapy can be successful if, even with large initial bacterial populations, there are feasible phage doses that will result in a bacteria levels decreasing to a small fraction of pre-treatment levels.

Because we assume that bacteria grow exponentially, rather than, say, logistically as in [2] or controlled by nutrient limitation as in $[11,10]$, there is no "phage-free" equilibrium with host cells at some positive level and no phage or infected cells. 


$$
\begin{aligned}
& \text { If } \frac{b N}{c}>a-p \text { and } \\
& \qquad \begin{aligned}
& (a-p) \int_{0}^{\tau} e^{-p s} \eta(s) d s>b V_{I}\left(1-(a-p) \tau \frac{\left(1-e^{-p \tau}\right)}{p \tau}\right)
\end{aligned}
\end{aligned}
$$

where the quotient $\frac{\left(1-e^{-p \tau}\right)}{p \tau}=1$ when $p=0$, then there exists a unique positive steady state $(X, Y, V)$. The analytical determination of the stability properties of the positive steady state via linearization is very challenging. See Beretta and Kuang [2] for some partial results. Simulations of our system extending for hundreds of hours duration, not shown here, are oscillatory in nature and characterized by long periods with cell densities well below one cell per milliliter. We conclude that the asymptotic behavior of the model system has limited relevance for phage growth.

\subsection{Parameter Values for Simulations}

Measurements of Heinemann et al. [23] found that the adsorption rate for phage T4 growing on an E. coli strain averaged $1.5 \times 10^{-8} \mathrm{ml} / \mathrm{min}$ which translates to the per hour rate in Table 1 . Similar values can be found in $[14,20]$.

Phillips et al. [4] observe that the waiting time following phage binding to host for initiation of injection is random, ranging from seconds to minutes. Their data reasonably fit an increasing but saturating exponential function with time constant $t_{0}$ which ranges from 79 seconds to 166 seconds. As they find that the injection process takes roughly 10 seconds, it is reasonable to take our injection time $1 / \rho$ to be 2 minutes.

This leads to a value $c=b / \rho=3 \times 10^{-8} \mathrm{ml}$ which means that the term $c v$ appearing in (1) is significant, i.e., $c v=O(0.1)$ when $v>0.3 \times 10^{7}$. In the numerical simulation shown in Figure 2, we take $v(0)=10^{8}$ (compare with Payne and Jansen [14] who use even $10^{9}$ initial phage in simulations) so $c v(0)=3$.

In his monograph, Stent [20] describes experiments of Schlesinger who measured the "adsorption capacity" of E. coli for WLL phage by adding phage to a suspension of bacteria and noting when additional phage could no longer become attached. He found that this threshold occurred at about 300 phage per bacterium. Although the adsorption capacity may differ from the number of binding sites for a variety of reasons, the experiment suggests that the number of binding sites satisfies $N=O\left(10^{2}\right)$. The lack of sensitivity 


\begin{tabular}{|c|c|}
\hline parameter & value \\
\hline $\mathrm{a}$ & $0.3 / \mathrm{hr}$ \\
$\mathrm{b}$ & $9 \times 10^{-7} \mathrm{ml} / \mathrm{hr}$ \\
$\mathrm{c}$ & $3 \times 10^{-8} \mathrm{ml}$ \\
$\mathrm{L}$ & 150 \\
$\mathrm{~m}$ & $1.8 / \mathrm{hr}$ \\
$\mathrm{p}$ & 0 \\
$\tau$ & $.43 \mathrm{hr}$ \\
$\mathrm{N}$ & 100 \\
$\rho$ & $30 / \mathrm{hr}$ \\
\hline
\end{tabular}

Table 1: System parameters

of the attack rate (1) to $N$ suggests that $N=100$ should give sufficient accuracy.

Parameter values used in our simulations are displayed in Table 1. Those not described above were taken from $[14,23]$. Our simulations are restricted to the special case that a burst of $L$ phage are produced from an infected cell at lysis. Therefore, equations (7) and (8) were used. Initial data were taken following Payne and Jansen.

Assuming that $U_{0}(s) \equiv 0$, the simulated system is:

$$
\begin{aligned}
\frac{d x}{d t} & =a x-\frac{b x v}{F_{N}(c v)}-p x \\
\frac{d y}{d t} & =\frac{b x v}{F_{N}(c v)}-H(t-\tau) e^{-p \tau} \frac{b x_{\tau} v_{\tau}}{F_{N}\left(c v_{\tau}\right)}-p y \\
\frac{d v}{d t} & =H(t-\tau) L e^{-p \tau} \frac{b x_{\tau} v_{\tau}}{F_{N}\left(v_{\tau}\right)}-b(x+y) v-m v
\end{aligned}
$$

where $H(t-\tau)$ denotes the heaviside function and $y(0)=0$ (reflecting that $\left.U_{0} \equiv 0\right)$ unless mentioned otherwise. Numerical solutions were computed using the dde23 delay differential equation solver on Matlab.

Thick lines in Figure 2 displays the time series simulating phage therapy over an hour time frame. A notable feature of the time series is the sharp discontinuity in the derivative of solution components at the time of the first latent period, roughly 26 minutes or 0.43 hours, caused by the burst of fresh phage. Cell concentrations below one cell per milliliter should be viewed 


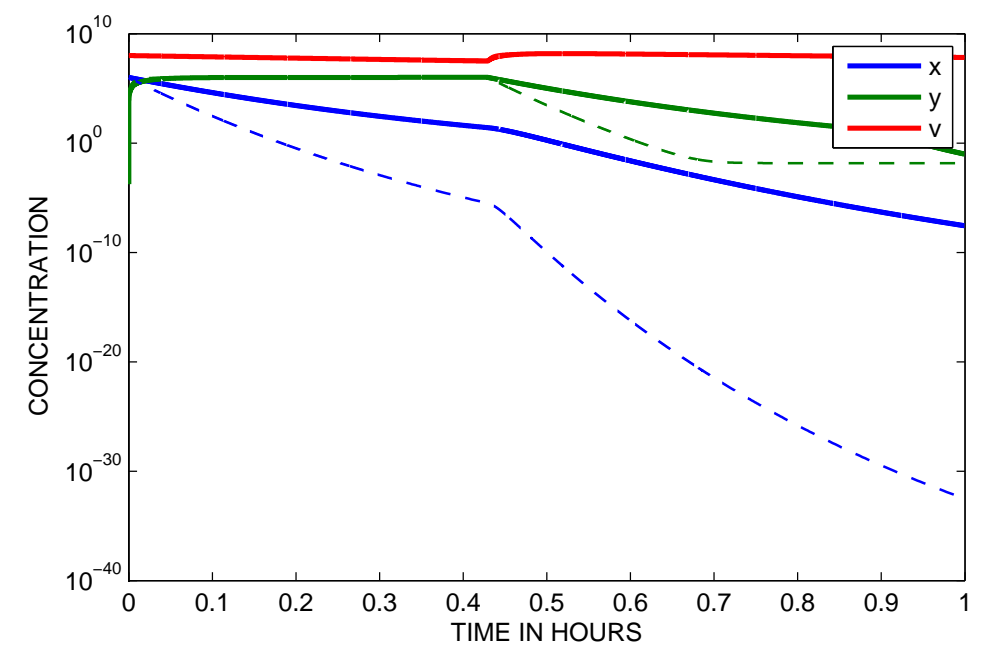

Figure 2: $x(0)=10^{6}, y(0)=0, v(0)=10^{8}$. Dashed lines correspond to $c=0$.

as the absence of cells; this occurs for uninfected cells at approximately 0.6 hours. For comparison purposes, the dashed lines show the result of replacing both the attack rate (1) and rate of phage loss (2) by the traditional mass action term $b x v$, keeping initial data the same. Note that the traditional mass-action rate results in a substantially quicker reduction in uninfected host over the first two latent periods and a reduction in infected cells over the second latent period. Uninfected cells essentially vanish at 0.2 hours and infected cells at 0.6 hours. Virus levels appear to be affected to a lesser degree. We conclude that the effect of our modifications in phage attack rate and phage loss rate is to significantly lengthen host cell survival, at least for initial data used here. As viral densities appear to be less affected by our modifications, it is probably the case that the modified attack rate is most significant.

\section{Implications for Phage Therapy}

Payne and Jansen $[14,15]$ discovered some key pharmacokinetic parameters related to in vivo phage therapy against bacterial infection using a very simple model of phage growth. Of course, effects of an immune response to phage and to bacteria are ignored for these calculations, assuming that they kick 
in at a later time.

As noted above, in the context of our deterministic model, successful phage therapy can at best mean that bacterial levels are driven to a sufficiently low level that the immune system easily finishes them off. Successful phage therapy requires at minimum that uninfected bacterial density decreases. Our equations imply that

$$
x^{\prime}<0 \Leftrightarrow v>V_{I}
$$

where $V_{I}$ is the unique positive root of $\frac{b v}{F_{N}(c v)}=a-p$ (see Lemma 1). Payne and Jansen [14] refer to their $V_{I}$, obtained by setting $c=0$ in ours, as the "inundation threshold" value. For the parameter values in Table $1, V_{I}=$ $3.3 \times 10^{5}$.

Note that there is no guarantee that if one starts out with (11) holding that it will continue to hold. In fact, Proposition 1 implies that $x^{\prime}<0$ cannot hold indefinitely. Therefore, merely arranging for initial phage densities to exceed the inundation threshold does not ensure "successful treatment".

Payne and Jansen identify two phage therapy strategies: passive therapy and active therapy. Passive therapy is the attempt to substantially knock down the bacterial population with the initial dose of phage, ignoring contributing effects of subsequent phage generations. Active therapy, presumably requiring a much smaller initial dose of phage, relies on the proliferation potential of phage reproduction to build up phage densities to levels sufficient to eventually drive down bacterial levels.

\subsection{Passive Therapy}

Payne and Jansen's derivation of an explicit minimal phage dose for successful passive therapy, i.e. reaching $x(t)=O(1)$, in [14] relies on the simplicity of their model. Indeed, there are excellent reasons for simple models and the ability to perform explicit calculations is one of them. Our model, which includes additional features such as the unproductive loss of phage due to phage attacking uninfected bacteria that are already bound to phage and to wasted phage attacks on infected cells, does not permit easy calculations. These additional features are most likely to be non-negligible at the required large phage doses used in passive therapy.

It is not clear that the notion of minimal dose for passive therapy is welldefined for our model. Here, we take passive therapy to mean that bacterial 
density is reduced to a suitably small fraction of its initial size within the initial latent period. We take this obviously restrictive view of passive therapy only for analytical convenience; it is not, however, at great variance from the rather subjective definition of passive therapy-not to rely on followon generations of phage for success.

It seems obvious on biological grounds that if we fix the initial uninfected bacterial density $x(0)$ and assume as usual that $y(0)=0$ but vary the initial phage dose $v(0)$, then larger doses will lead to smaller uninfected bacterial levels. However, this is far from obvious from a mathematical viewpoint. The next result establishes this point and provides a theoretical basis for the concept of minimal dose for passive therapy.

Proposition 2. Let $(x(t), y(t), v(t))$ and $(\bar{x}(t), \bar{y}(t), \bar{v}(t))$ be two solutions of (10) with $x(0)=\bar{x}(0)$ and $y(0)=\bar{y}(0)=0$. If $v(0)<\bar{v}(0)$ then $\bar{x}(t)<x(t)$ and $\bar{x}(t)+\bar{y}(t)<x(t)+y(t)$ on $0<t \leq \tau$.

For every $\theta \in(0,1)$ and every $U>0$ there exists $V=V(U)>0$ such that if $(x(t), y(t), v(t))$ is a solution of $(10)$ with $x(0)+y(0) \leq U$ and $v(0) \geq V$, then

$$
\frac{x(\tau)}{x(0)} \leq e^{(a-p-(b N / c) \theta) \tau}
$$

In words, bigger phage doses result in smaller bacteria levels over the first latent period. In addition, for any initial bacterial density, there is a phage dose which will reduce bacterial density at the end of the first latent period by a factor that can be taken as close to $e^{(a-p-(b N / c) \tau}$ as desired. Recall that we are assuming $a-p-(b N / c)<0$. Indeed, for the parameters of Table 1 it is approximately -1290 . Therefore, $e^{(a-p-(b / c) \theta) \tau} \approx \exp (-1290)$ if $\theta \approx 1$. We do not claim that the required doses are medically feasible or even that there are the required number of phage in the universe. The proof of Proposition 2 is provided in the appendix.

\subsection{Active Therapy}

In their consideration of active treatment, Payne and Jansen give an intuitive argument for the existence of a threshold bacterial density such that phage numbers are amplified over each successive phage generation only if bacterial density exceeds threshold. We can apply this intuitive reasoning to our model as well although we arrive at a somewhat different threshold for phage amplification. According to our delay model, a cohort of $y_{0}$ newly infected cells, 
i.e. $U_{0}(s)=y_{0} \delta(s)$ where $\delta$ is the Dirac impulse concentrated at zero so all cells have infection-age zero, gives rise to $y_{0} L e^{-p \tau}$ phage $\tau$ units of time later when the surviving members of this cohort of infected host have lysed. These phage survive $\frac{1}{b(x+y)+m}$ hours during which they infect host at rate $\frac{b x}{F_{N}(c v)}$ per phage. We conclude that the $y_{0} L e^{-p \tau}$ phage produce $L e^{-p \tau} \frac{b x}{(b(x+y)+m) F_{N}(c v)} y_{0}$ second generation infected cells. The amplification factor (of $\left.y_{0}\right)$ is therefore

$$
R_{0}=L e^{-p \tau} \frac{b x}{(b(x+y)+m) F_{N}(c v)}
$$

The condition for amplification of infected cells, and hence, the condition for proliferation of phage is that $R_{0}>1$ :

$$
L e^{-p \tau} \frac{b x}{(b(x+y)+m) F_{N}(c v)}>1
$$

Unlike the proliferation threshold derived in [14], ours does not lead to a threshold condition for uninfected bacteria alone. However, as active therapy should not require such large phage doses that $c v$ is significant, it may be reasonable in some cases to assume $c v \ll 1$ in which case $F_{N}(c v) \approx 1$ and we may ignore this factor. For parameter values in Table one, if $v<10^{7}$ this is a good approximation. Immediately below, we assume this approximation is valid.

Our proliferation condition involves both infected and uninfected host. If $b(x+y)$ is small relative to $m$, we arrive at a threshold bacterial density for phage proliferation that is comparable to the one obtained in [14] and corrects the one given in [23]:

$$
x>X_{p} \approx \frac{m}{b L e^{-p \tau}}
$$

However, if $b(x+y)$ is large relative to $m$, then (13) yields a threshold on the fraction of uninfected cells

$$
L e^{-p \tau} \frac{x}{x+y}>1
$$

In summary, the proliferation threshold computed from our model is more complex than that of Payne and Jansen since it involves all three quantities $x, y, v$. If $c v \ll 1$, it reduces to one similar to theirs, a lower bound on uninfected bacteria, when total bacterial density is not too large but gives a 
lower bound for the fraction of uninfected bacteria when the total bacterial density is large. For parameter values in Table $1, \frac{m}{b L e^{-p \tau}} \approx 13,500 ; b(x+y) \ll$ $m$ if $x+y<10^{4}$.

We provide two simulations where active therapy can be clearly identified. Initial data in Figure 4 yield a value $R_{0} \approx 7$ well above unity. Bacteria initially grow before phage gain control after the initial latent period. Figure 5 can be compared to Payne and Jansen's Figure 1(c) in [14] which shows passive therapy. We chose parameter values to correspond to those in that figure: $a=0.3, b=10^{-6}, \tau=0.83, L=100, m=1.8, p=0$ with our value of $N$ and $c=b / \rho=0.33 \times 10^{-7}$. Initial data were chosen as in their figure as well except that our starting time corresponds to their $t_{\phi}$. It must be kept in mind that our model differs from theirs in the attack rate as well as our assumption that infected host do not grow. Our simulation agrees qualitatively with theirs although our peak bacterial density exceeds theirs by a factor of three.

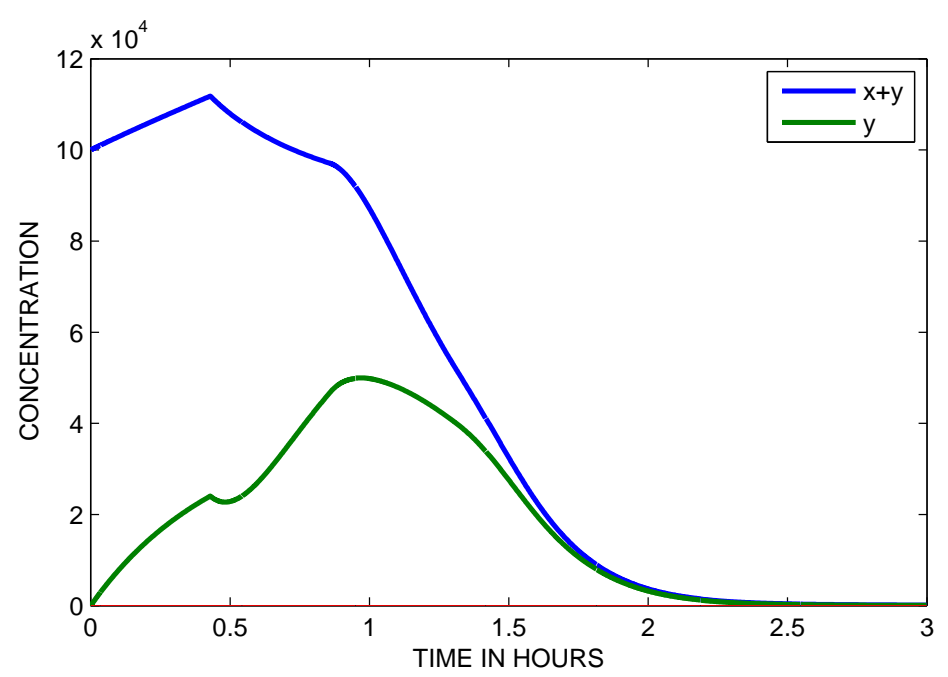

Figure 3: Active therapy with $x(0)=10^{5}, v(0)=10^{6}$.

\subsection{Summary of Conclusions for Phage Therapy}

We have explored a mathematical model of virulent phage growth on an exponentially growing bacterial population, system (10), which differs from previous models in two ways: (i) the density-dependent phage attack rate 


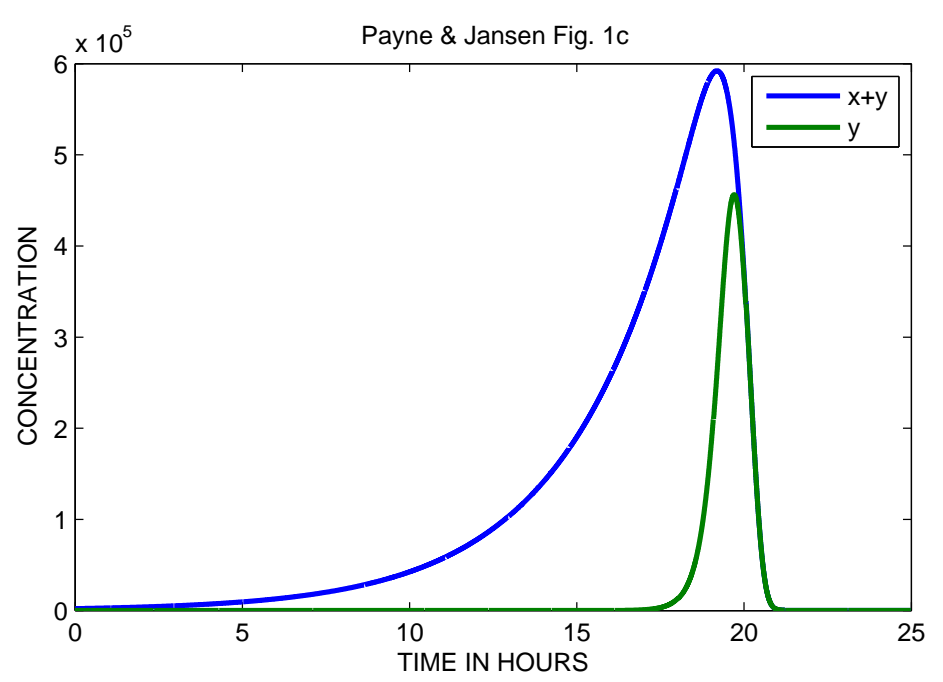

Figure 4: Compare to Payne and Jansen Figure 1(c): $x(0)=2117, v(0)=$ 100

(1) is used in place of the mass action rate, and (ii) the loss rate of phage due to attachment (2) includes all bacterial cells not just uninfected ones. Our simulations, particularly Figure 2, appear to be most strongly influenced by the modification (i), leading to significantly lengthened host cell survival. However, (ii) played a role in the calculation of the proliferation threshold (13) for active therapy which has a more complicated character than the simpler one deduced in [14]. Finally, in Proposition 2 we established that passive therapy works: for any initial bacterial population there is a phage dose that can reduce the host population to an insignificant fraction of its initial level within the first latent period. Although the dose may be impractically large and the restriction to a single latent period unduly restrictive, our result establishes the principle. It remains to be seen whether any of these effects are important for phage therapy.

In the following appendix, we show how (i) and (ii) arise from a careful modeling of bacteria-phage complexes consisting of a single host and a number of attached phage. The modeling framework we introduce there may be more important in the long run than the conclusions described above since it leads to much more flexibility in modeling phage release and host cell survival. 


\section{Appendix}

We explore several modeling issues in this appendix which may be of interest for general bacteriophage-host interactions. First, we construct a model where infected host are structured by injection-age and where various complexes consisting of a host cell and one or more attached phage are explicitly included. We assume that each cell has a fixed number $N$ of binding sites and where host cells can have multiple attached phage. Finally, the agestructured infected cell population is reduced to integro-differential equations. Two cases lead to relatively simple equations: (1) the case where infected cell death rate by lysis is zero until time $\tau$ after which it is infinite, equivalent to fixed-length latent period, and (2) the lysis rate is constant meaning an exponentially distributed latent period. The first case leads to the model considered in previous sections while the second case leads to simpler ordinary differential equations similar to ones in [14].

\subsection{Phage-Host Complex Formation}

We call a host cell infected when a phage has injected genetic material into it; until then it is called uninfected. Furthermore, a phage ceases to exist once it has injected its genetic material.

The time between injection and lysis is on the order of 20 minutes or so, depending on the host-phage system. We will keep track of this "infection age" of infected cells. Denote by $Y(t, s)$ the distribution of infected cells of age $s$ at time $t$. The integral

$$
\int_{a_{1}}^{a_{2}} Y(t, s) d s
$$

then gives the number of infected (post-injection) cells that were injected between $t-a_{1}$ and $t-a_{2}$ and

$$
Y=\int_{0}^{\infty} Y(t, s) d s
$$

gives the size of the infected class of cells.

Assume that each host cell has $N$ potential phage binding sites. Then we 
partition uninfected host $X$, infected host $Y$, and phage $V$ as follows:

$$
\begin{aligned}
X(t) & =\sum_{i} C_{x}^{i}(t) \\
Y(t, s) & =\sum_{i} C_{y}^{i}(t, s) \\
V(t) & =v(t)+\sum_{i} i\left(C_{x}^{i}(t)+\int_{0}^{\infty} C_{y}^{i}(t, s) d s\right)
\end{aligned}
$$

$C_{x}^{i}\left(C_{y}^{i}\right)$ denotes the concentration of uninfected (infected) cells having $i$ attached phage, for $0 \leq i \leq N$, and $v(t)$ denotes the concentration of unadsorbed phage. In the case of infected cells, we stress that the age variable $s$ denotes time since injection, not time in a particular compartment. All sums are over the range $0 \leq i \leq N$.

Recall that a host cell is infected once an attached phage injects and a phage ceases to exist once it injects.

We assume that a host complex with $i<N$ attached phage may adsorb an additional phage at the rate $b v C_{z}^{i}$ where $z=x, y$. The rate constant $b$, the adsorption rate, is assumed independent of $i$ and whether the host complex is infected $(z=y)$ or uninfected $(z=x)$. Let $\nu(s)$ denote the death (lysis) rate of infected cells of age $s$ and let $\eta(s)$ denote the rate of release of phage from an infected cell of age $s$. Here, we explore the case that both are independent of the number of attached phage. Recall that $\rho$ is the injection rate, equivalently, $1 / \rho$ is the average time between phage binding and subsequent injection of genetic material. The model equations are as 
follows:

$$
\begin{aligned}
X^{\prime} & =a X-\rho \sum_{i=1}^{N} i C_{x}^{i}-p X \\
\left(C_{x}^{i}\right)^{\prime} & =a C_{x}^{i}+b v C_{x}^{i-1}-(i \rho+b v) C_{x}^{i}-p C_{x}^{i} \\
\left(\frac{\partial}{\partial t}+\frac{\partial}{\partial s}\right) C_{y}^{i}(t, s) & =b C_{y}^{i-1} v+(i+1) \rho C_{y}^{i+1}-(i \rho+b v) C_{y}^{i}-(p+\nu(s)) C_{y}^{i} \\
C_{y}^{i}(t, 0) & =\rho(i+1) C_{x}^{i+1}(t) \\
\left(\frac{\partial}{\partial t}+\frac{\partial}{\partial s}\right) Y(t, s) & =-(\nu(s)+p) Y(t, s) \\
Y(t, 0) & =\rho \sum_{i=1}^{N} i C_{x}^{i}(t) \\
v^{\prime} & =\int_{0}^{\infty} Y(t, s) \eta(s) d s-m v \\
& -b v \sum_{i=0}^{N-1}\left(C_{x}^{i}+\int_{0}^{\infty} C_{y}^{i}(t, s) d s\right)
\end{aligned}
$$

where $C_{x}^{N+1}=C_{y}^{N+1}=0$ and where for $i=N$, the loss term $-b v C_{z}^{N}, z=x, y$ in the equations for $C_{z}^{N}$ is dropped since all binding sites are filled so no more attachments are allowed.

Some comments are in order as these equations may not at first appear transparent. Total uninfected host are lost due to injection of a complex $C_{x}^{i}(t)$ by one of its attached phage, which then becomes a newly infected host $C_{y}^{i-1}(t, 0)$ with one less attached phage. The rate of injection is $\rho i C_{x}^{i}$ since any one of the $i$ attached phage may inject. This accounts for the loss term $-i \rho C_{x}^{i}(t)$ in the equation for $X$ and the boundary condition for $C_{y}^{i}(t, s)$ and $Y(t, s)$ at $s=0$. The injection of an infected host simply reduces the number of attached phage by one. Figure 6 shows the flow between the compartments $C_{x}^{i}$ and $C_{y}^{i}$. Free phage are lost due to attachment to host cells.

Initial conditions for (15) consist of specifying nonnegative values for $X(0), C_{x}^{i}(0), v(0)$ and nonnegative functions $C_{y}^{i}(0, s)$ and $Y(0, s)=U_{0}(s)$ defined for $s \geq 0$.

We intend to employ a quasi-steady state analysis in order to remove the equations for the host/phage complexes. Before doing so it is convenient to 

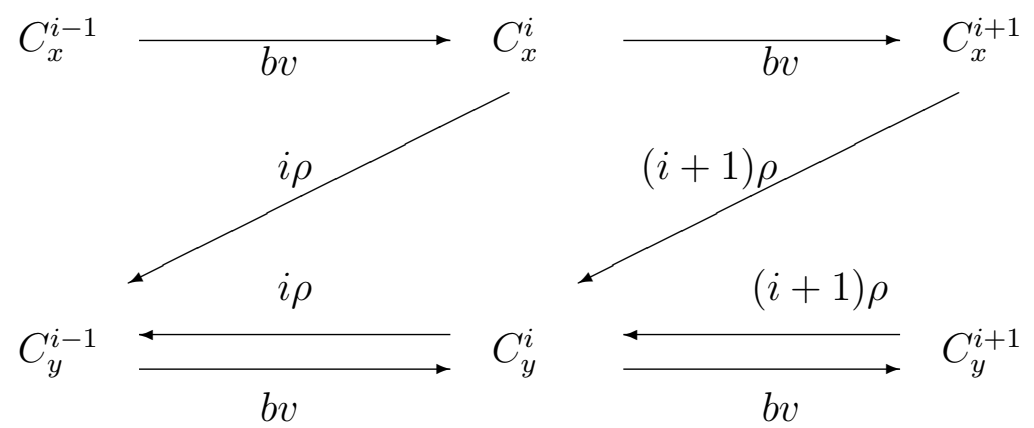

Figure 5: Transfer Diagram between Complexes for equations (15): $\rho$ denotes injection rate, $b v$ denotes adsorption rate.

integrate the equation for $C_{y}^{i}$ with respect to infection age, yielding $\left(C_{y}^{i}\right)^{\prime}=-\int_{0}^{\infty}(\nu(s)+p) C_{y}^{i}(t, s) d s-(i \rho+b v) C_{y}^{i}+b v C_{y}^{i-1}+(i+1) \rho\left(C_{y}^{i+1}+C_{x}^{i+1}\right)$

Hereafter, unless specifically mentioned, $C_{y}^{i}$ denotes

$$
C_{y}^{i}(t)=\int_{0}^{\infty} C_{y}^{i}(t, s) d s
$$

We assume that irreversible binding and injection are fast compared to such processes as growth and washout of host and the latent period. Therefore we ignore these slower processes in the equations for complexes $C_{x}^{i}$ and $C_{y}^{i}$ in (15). Setting the time derivatives to zero in the $C_{x}^{i}$ and $C_{y}^{i}$ equations yields:

$$
\begin{aligned}
& 0=b v C_{x}^{i-1}-(i \rho+b v) C_{x}^{i}, 1 \leq i \leq N \\
& 0=b v C_{y}^{i-1}+(i+1) \rho\left(C_{x}^{i+1}+C_{y}^{i+1}\right)-(i \rho+b v) C_{y}^{i}
\end{aligned}
$$

where we employ the conventions used in (15).

Adding the first $N$ equations for the $C_{x}^{i}$ and then adding all $2 N$ equations yields

$$
\sum_{i=1}^{N} i C_{x}^{i}=b v C_{x}^{0} / \rho, \quad C_{x}^{1}+C_{y}^{1}=b v\left(C_{x}^{0}+C_{y}^{0}\right) / \rho
$$

Then straightforward calculations give the distribution of phage occupancy of host cell binding sites:

$$
C_{x}^{i}+C_{y}^{i}=\frac{(b v / \rho)^{i}}{i !}\left(C_{x}^{0}+C_{y}^{0}\right)
$$


meaning phage are distributed according to a Poisson distribution with parameter (mean and variance) $b v / \rho$. We also have

$$
C_{x}^{i}=C_{x}^{0} \prod_{j=1}^{i} \frac{u}{j+u}, 1 \leq i \leq N-1, C_{x}^{N}=\frac{u}{N} C_{x}^{N-1}, u=b v / \rho
$$

We must express all quantities in terms of the variables $X, Y, v$ used in equations (15). We summarize the results of doing so below.

Proposition 3. The phage attack rate is

$$
-\rho \sum_{i=1}^{N} i C_{x}^{i}=-\frac{b v X}{F_{N}(c v)}, c=b / \rho
$$

where

$F_{N}(u)=1+\frac{u}{1+u}+\frac{u^{2}}{(1+u)(2+u)}+\cdots+\frac{u^{N}}{(1+u)(2+u) \cdots(N-1+u) N}$

The rate of loss of free phage due to attachment satisfies

$$
-b v \sum_{i=0}^{N-1}\left(C_{x}^{i}+C_{y}^{i}\right)=-b v(X+Y)\left(1-\frac{\frac{u^{N}}{N !}}{\sum_{i=0}^{N} \frac{u^{i}}{i !}}\right)
$$

where $Y=Y(t)=\int_{0}^{\infty} Y(t, s) d s$.

Proof. In order to relate $x$ to $X$ we use (17):

$$
\begin{aligned}
X= & \sum_{i} C_{x}^{i} \\
= & C_{x}^{0}\left(1+\frac{u}{1+u}+\frac{u^{2}}{(1+u)(2+u)}\right. \\
& \left.+\cdots+\frac{u^{N}}{(1+u)(2+u) \cdots(N-1+u) N}\right) \\
= & C_{x}^{0} F_{N}(u)
\end{aligned}
$$

Similarly, using (16),

$$
X+Y=\sum_{i=0}^{N}\left(C_{x}^{i}+C_{y}^{i}\right)=\left(C_{x}^{0}+C_{y}^{0}\right) \sum_{i=0}^{N} \frac{u^{i}}{i !}
$$


Finally,

$$
\begin{aligned}
\sum_{i=0}^{N-1}\left(C_{x}^{i}+C_{y}^{i}\right) & =X+Y-\left(C_{x}^{N}+C_{y}^{N}\right) \\
& =(X+Y)-\frac{u^{N}}{N !}\left(C_{x}^{0}+C_{y}^{0}\right) \\
& =(X+Y)\left(1-\frac{\frac{u^{N}}{N !}}{\sum_{i=0}^{N} \frac{u^{i}}{i !}}\right) \\
& \approx X+Y
\end{aligned}
$$

In view of the above result and the quasi-steady state analysis, our system (15) reduces to:

$$
\begin{aligned}
X^{\prime} & =a X-\frac{b v X}{F_{N}(c v)}-p X \\
\left(\frac{\partial}{\partial t}+\frac{\partial}{\partial s}\right) Y(t, s) & =-(\nu(s)+p) Y(t, s) \\
Y(t, 0) & =\frac{b v X}{F_{N}(c v)} \\
v^{\prime} & =\int_{0}^{\infty} Y(t, s) \eta(s) d s-m v \\
& -b v\left(X(t)+\int_{0}^{\infty} Y(t, s) d s\right)
\end{aligned}
$$

Our analysis above shows that the parameter $c$ in (1) is given by

$$
c=\frac{b}{\rho}=b \times \text { adsorption time }
$$

We may solve for $Y(t, s)$ by integrating along characteristics:

$$
Y(t, s)=\left\{\begin{array}{cc}
R(t-s) e^{-p s} e^{-\int_{0}^{s} \nu(u) d u}, & t>s \\
U_{0}(s-t) e^{-p t} e^{-\int_{s-t}^{s} \nu(u) d u}, & t<s
\end{array}\right\}
$$

where $R(t)=\frac{b v X}{F_{N}(c v)}$. The total infected cell population, $Y(t)=\int_{0}^{\infty} Y(t, s) d s$, is easily computed:

$$
Y(t)=\int_{0}^{t} R(t-s) e^{-p s} e^{-\int_{0}^{s} \nu(u) d u} d s+\int_{0}^{\infty} U_{0}(s) e^{-p t} e^{-\int_{s}^{s+t} \nu(u) d u} d s
$$


and similarly for the equation for phage:

$$
\begin{aligned}
v^{\prime}= & -b v(X+Y)-m v+\int_{0}^{t} R(t-s) e^{-p s} \eta(s) e^{-\int_{0}^{s} \nu(u) d u} d s \\
& +e^{-p t} \int_{0}^{\infty} U_{0}(s) \eta(s+t) e^{-\int_{s}^{t+s} \nu(u) d u} d s
\end{aligned}
$$

System (18) can now be replaced by the equation for $X$ together with the equations above for $Y$ and $v$. Below we consider two special cases for the lysis rate $\nu$.

\subsection{Fixed-Length Latent Period}

The special case

$$
\nu(s)=\left\{\begin{array}{cc}
0, & s<\tau \\
\infty, & s>\tau
\end{array}\right\}
$$

leads to

$$
e^{-\int_{0}^{s} \nu(u) d u}=\chi_{[0, \tau]}(s), e^{-\int_{s}^{s+t} \nu(u) d u}=\left\{\begin{array}{ll}
1, & s+t<\tau \\
0, & s+t>\tau
\end{array}\right\}
$$

where, for a set $B, \chi_{B}(z)=1$ if $z \in B$, otherwise $\chi_{B}(z)=0$.

Hence

$$
\begin{aligned}
Y(t) & =\int_{0}^{\min \{t, \tau\}} R(t-s) e^{-p s} d s+\int_{0}^{\infty} U_{0}(s) e^{-p t} \chi_{\{s+t<\tau\}}(t, s) d s \\
& =\int_{0}^{\min \{t, \tau\}} R(t-s) e^{-p s} d s+\chi_{[0, \tau]}(t) \int_{0}^{\tau-t} U_{0}(s) e^{-p t} d s
\end{aligned}
$$

Equivalently,

$$
Y(t)=\left\{\begin{array}{cc}
\int_{0}^{t} R(t-s) e^{-p s} d s+\int_{0}^{\tau-t} U_{0}(s) e^{-p t} d s, & t<\tau \\
\int_{0}^{\tau} R(t-s) e^{-p s} d s, & t>\tau
\end{array}\right\}
$$

The equation for phage becomes

$v^{\prime}=-b v(X+Y)-m v+\int_{0}^{\min \{\tau, t\}} R(t-s) e^{-p s} \eta(s) d s+e^{-p t} \chi_{[0, \tau]}(t) \int_{0}^{\tau-t} U_{0}(s) \eta(s+t) d s$ 
Equivalently,

$$
v^{\prime}=\left\{\begin{array}{cc}
-b v(X+Y)-m v+\int_{0}^{t} R(t-s) e^{-p s} \eta(s) d s+e^{-p t} \int_{0}^{\tau-t} U_{0}(s) \eta(s+t) d s, & t<\tau \\
-b v(X+Y)-m v+\int_{0}^{\tau} R(t-s) e^{-p s} \eta(s) d s, & t>\tau
\end{array}\right\}
$$

If

$$
\eta(s)=L \delta(s-\tau)
$$

Then

$$
v^{\prime}=\left\{\begin{array}{ll}
-b v(X+Y)-m v+L e^{-p t} U_{0}(\tau-t), & t<\tau \\
-b v(X+Y)-m v+L R(t-\tau) e^{-p \tau}, & t>\tau
\end{array}\right\}
$$

These, together with the equation for $X$ from (18), are the equations considered in section 2 .

\subsection{Exponentially Distributed Latent Period}

In case of an exponentially distributed latent period, $\nu(s) \equiv \nu$, easy computations yield expressions for the infected:

$$
Y(t)=\int_{0}^{t} R(s) e^{-(p+\nu)(t-s)} d s+e^{-(p+\nu) t} \int_{0}^{\infty} U_{0}(s) d s
$$

or, on differentiation,

$$
Y^{\prime}=-(p+\nu) Y+R(t), Y(0)=\int_{0}^{\infty} U_{0}(s) d s
$$

The phage equation becomes:

$v^{\prime}=-b v(X+Y)-m v+\int_{0}^{t} R(s) \eta(t-s) e^{-(p+\nu)(t-s)} d s+e^{-(p+\nu) t} \int_{0}^{\infty} U_{0}(s) \eta(s+t) d s$

If $\eta(s) \equiv L \nu$ then $v$ satisfies:

$$
\begin{aligned}
v^{\prime} & =-b v(X+Y)-m v+\nu L\left(\int_{0}^{t} R(s) e^{-(p+\nu)(t-s)} d s+e^{-(p+\nu) t} \int_{0}^{\infty} U_{0}(s) d s\right) \\
& =-b v(X+Y)-m v+\nu L y
\end{aligned}
$$

These, together with the equation for $X$ from (18), can be compared to those of Payne and Jansen [14]. 


\subsection{Proof of Lemma 1}

Straightforward calculation yield that

$$
F_{N+1}(u)=F_{N}(u)-\frac{u^{N+1}}{(1+u) \cdots(N+u) N(N+1)}
$$

and that $F_{N}(u) / u \rightarrow 1 / N$ as $u \rightarrow \infty$.

We show that $\frac{u}{F_{N}(u)}$ is monotonically increasing by showing it has a positive derivative. Since we may write

$$
\frac{u}{F_{N}(u)}=\frac{u}{F_{1}(u)} \frac{F_{1}(u)}{F_{2}(u)} \cdots \frac{F_{N-1}(u)}{F_{N}(u)}
$$

it suffices to show that $\frac{u}{F_{1}(u)}$ and $\frac{F_{n}(u)}{F_{n+1}(u)}$ have positive derivatives for $n \geq 1$. $\frac{u}{F_{1}(u)}=u / 1+u$ is clearly increasing.

$$
\begin{aligned}
\frac{d}{d u} \frac{F_{n}(u)}{F_{n+1}(u)} & =\frac{d}{d u} \frac{F_{n+1}(u)+g(u)}{F_{n+1}(u)} \\
& =\frac{g(u)}{u F_{n+1}(u)}\left(\frac{u g^{\prime}(u)}{g(u)}-\frac{u F_{n+1}^{\prime}(u)}{F_{n+1}(u)}\right)
\end{aligned}
$$

where

$$
g(u)=\frac{u^{n+1}}{(1+u)(2+u) \cdots(n+u)(n+1) n}
$$

Straightforward computation gives:

$$
\frac{u g^{\prime}(u)}{g(u)}=1+\sum_{i=1}^{n} \frac{i}{i+u}
$$

and

$$
\begin{aligned}
u F_{n+1}^{\prime}(u)= & \frac{u}{1+u}\left(\frac{1}{1+u}\right)+\frac{u^{2}}{(1+u)(2+u)}\left(\frac{1}{1+u}+\frac{2}{2+u}\right)+\cdots \\
& +\frac{u^{n}}{(1+u)(2+u) \cdots(n+u)}\left(\frac{1}{1+u}+\frac{2}{2+u}+\cdots+\frac{n}{n+u}\right) \\
& +\frac{u^{n+1}}{(1+u)(2+u) \cdots(n+u)(n+1)}\left(1+\frac{1}{1+u}+\frac{2}{2+u}+\cdots+\frac{n}{n+u}\right)
\end{aligned}
$$


On dividing this expression by $F_{n+1}(u)$ we see that it can be viewed as a convex combination of the quantities in parentheses in the previous expression and therefore it lies between the minimum and maximum of the quantities in parentheses. But the maximum is clearly inside the last parenthesis which exactly agrees with $\frac{u g^{\prime}(u)}{g(u)}$. We conclude that

$$
\frac{u g^{\prime}(u)}{g(u)}-\frac{u F_{n+1}^{\prime}(u)}{F_{n+1}(u)}>0
$$

\subsection{Proof of Proposition 2}

Setting $u=x+y$ in our system (10), we obtain the system

$$
\begin{aligned}
& \frac{d x}{d t}=a x-\frac{b x v}{F_{N}(c v)}-p x \\
& \frac{d u}{d t}=a x-p u, \quad 0 \leq t \leq \tau \\
& \frac{d v}{d t}=-b u v-m v
\end{aligned}
$$

This is a monotone system of differential equations whose forward flow preserves the order relation

$$
(\bar{x}, \bar{u}, \bar{v}) \leq_{K}(x, u, v) \Leftrightarrow \bar{x} \leq x, \bar{u} \leq u, v \leq \bar{v}
$$

See Chapt.3, sec. 5 of [19]. As the Jacobian matrix of the right hand side is irreducible, it follows that for $0<t \leq \tau$

$(\bar{x}(0), \bar{u}(0), \bar{v}(0))<_{K}(x(0), u(0), v(0)) \Rightarrow(\bar{x}(t), \bar{u}(t), \bar{v}(t)) \ll_{K}(x(t), u(t), v(t))$

where $<_{K}$ means at least one strict inequality in (20) while $\ll_{K}$ means all inequalities are strict. The first assertion of Proposition 2 is an immediate consequence of the previous inequality.

As for the second assertion of Proposition 2, we need some elementary estimates. Inequality $u^{\prime} \leq(a-p) u$ leads to $u(t) \leq u(0) e^{(a-p) t}$ so there exists $M \geq 1$ such that $u(t) \leq M u(0), 0 \leq t \leq \tau$. Similarly, $v^{\prime} \geq-[b M u(0)+m] v$ leads to $v(t) \geq K v(0), 0 \leq t \leq \tau$ where $K=e^{-(b M u(0)+m) \tau}$. If $0<\theta<1$, then

$$
\frac{c v}{F_{N}(c v)} \geq \frac{c K v(0)}{F_{N}(c K v(0))} \geq \theta N
$$

by taking $v(0)$ sufficiently large. Hence, $\frac{x^{\prime}}{x} \leq a-p-(b N / c) \theta$. 


\section{References}

[1] S. Abedon, T. Herschler, D. Stopar, Bacteriophage Latent-Period Evolution as a Response to Resource Availability, Appl. \& Environ. Microbiol. 67(2001), 4233-4241.

[2] E. Beretta and Y. Kuang, Modeling and analysis of a marine bacteriophage infection with latency, Nonlinear Analysis RWA 2(2001) 35-74.

[3] A. Campbell, Conditions for the existence of bacteriophage, Evolution 15 (1961) 153-165.

[4] P. Grayson, L. Han, T. Winther, R. Phillips, Real-time observations of single bacteriophage lambda DNA ejection in vitro, PNAS 104, no. 37 (2007), 14652-57.

[5] G. Gripenberg, S-O.Londen, \& O Staffans, Volterra Integral and Functional Equations, Encyclopedia of Mathematics and its Applications, Cambridge Univ. Press, Cambridge 1990.

[6] T. Håusler, Viruses vs. Superbugs, a solution to the antibiotic crisis?, MacMillan, New York 2006.

[7] L. Kasman,A. Kasman,C. Westwater,J. Dolan,M. Schmidt, J. Norris, Overcoming the phage proliferation threshold, a mathematical model with implications for phage therapy, J. Virol. 76 (2002) 5557-5564.

[8] L. Kasman,A. Kasman,C. Westwater,J. Dolan,M. Schmidt, J. Norris, Letter to the editor, Author's reply, J. Virol. 76 (2002) 13123.

[9] B. Levin, F. Stewart, L. Chao, Resource-limited growth, competition, and predation: a model and experimental studies with bacteria and bacteriophage, Amer. Nat. 111(1977), 3-24.

[10] R. Lenski and B. Levin, Constraints on the coevolution of bacteria and virulent phage: a model, some experiments, and predictions for natural communities, Amer. Naturalist 125(4), 1985, 585-602.

[11] B. Levin, J. Bull, Phage therapy revisited: the population biology of a bacterial infection and its treatment with bacteriophage and antibiotics, the American Naturalist 147, 1996, 881-898. 
[12] B. Levin, J. Bull, Population and Evolutionary Dynamics of Phage Therapy, Nature Reviews Microbiology, 2 (2004) 166-173.

[13] S. Matsuzaki, M. Rashel, J.Uchiyama, S. Sakurai, T.Ujihara, M.Kuroda, M Ikeuchi, T.Tani, M.Fujieda, H.Wakiguchi, S.Imai, Bacteriophage therapy: a revitalized therapy against bacterial infectious diseases, J. Infect. Chemother. 11(2005) 211-219.

[14] R. Payne, V. Jansen, Understanding Bacteriophage Therapy as a density-dependent kinetic process, J. Theor. Biol. 208 (2001), 37-48.

[15] R. Payne and V. Jansen, Pharmacokinetic Principles of Bacteriophage Therapy, Clin. Pharmacokinetics 42(4), 2003, 315-325.

[16] R. Payne and V. Jansen, Evidence for a phage proliferation threshold, Letter to the editor, J. Virol. 76 (2002) 13123.

[17] Z. Qui, The analysis and regulation for the dynamics of a temperate bacteriophage model, Mathematical Biosci., to appear.

[18] S. Schrag and J. Mittler, Host-Parasite Coexistence: the role of spatial refuges in stabilizing bacteria-phage interactions, Amer. Naturalist 148, 1996, 348-377.

[19] H.L. Smith, Monotone Dynamical Systems: an introduction to the theory of competitive and cooperative systems, Amer. Math. Soc. Surveys and Monograghs, 41, 1995.

[20] G. Stent, Molecular Biology of Bacterial Viruses, W.H. Freeman and Co., London, 1963.

[21] H. Thieme and J. Yang, On the Complex formation approach in modeling predator prey relations, mating, and sexual disease transmission, Elect. J. Diff. Eqns. 05, 2000, 255-283.

[22] J. Weitz,H. Hartman, and S. Levin, Coevolutionary arms race between bacteria and bacteriophage, Proc. Nat. Acad. Sci. 102, no 27, (2005) 9535-9540.

[23] R. Weld, C. Butts, J. Heinemann, Models of phage growth and their applicability to phage therapy, J. Theor. Biol. 227, (2004), 1-11. 